\title{
AGENT BASED IMAGE ANALYSIS (ABIA) - PRELIMINARY RESEARCH RESULTS FROM AN IMPLEMENTED FRAMEWORK
}

Peter Hofmann ${ }^{\mathrm{a}, *}$, Vera Andrejchenko ${ }^{\mathrm{a}}$, Paul Lettmayer ${ }^{\mathrm{b}}$, Manuel Schmitzberger ${ }^{\mathrm{b}}$, Michael Gruber ${ }^{\mathrm{b}}$, Izzet Ozan ${ }^{\mathrm{b}}$, Mariana Belgiu $^{\mathrm{a}}$, Roland Graf ${ }^{\mathrm{b}}$, Thomas Josef Lampoltshammer ${ }^{\mathrm{b}}$, Stefan Wegenkittl ${ }^{\mathrm{b}}$, Thomas Blaschke ${ }^{\mathrm{a}}$

anterfaculty Department of Geoinformatics - Z_GIS, Salzburg University, Schillerstr. 30, 5020 Salzburg, Austria. (*peter.hofmann,vera.andrejchenko,mariana.belgiu,thomas.blaschke)@ sbg.ac.at.

bepartment of Information Technology \& Systems Management, Salzburg University of Applied Sciences, Austria. (((mschmitzberger,mgruber,iozan).its-m2014,plettmayer.its-m2013),stefan.wegenkittl,roland.graf)@ fhsalzburg.ac.at,thomas.lampoltshammer@gmail.com

KEY WORDS: Agent Based Image Analysis, Object Based Image Analysis, Software Agents, Autonomous Systems, Adaptive Systems, Multi Agent Systems

\begin{abstract}
:
Object Based Image Analysis (OBIA) has meanwhile been established as a paradigm for analyzing remotely sensed image data. Although the degree of automation for OBIA methods has increased for several applications, especially in the domain of remote sensing, robust and transferable object-based solutions for automated image analysis of sets of images or even large image archives are still rare. One of the reasons for this lack of robustness and transferability is the high complexity of remote sensing image contents: Especially in Very High Resolution (VHR) remote sensing data, under varying imaging conditions or sensor characteristics, the objects' properties can vary unpredictably. Although earlier work has demonstrated that OBIA rule sets bear a high potential of transferability these rule sets need to be adapted manually in order to receive acceptable results, or the classification results need to be adjusted manually in a post-processing step. In order to automate these adaptation and adjustment procedures we investigate the coupling, extension and integration of OBIA with the agent-based paradigm, which is exhaustively investigated in software engineering and robotics. The aims of such integration are a) rule sets which can be adapted autonomously according to varying imaging data, and b) image objects which can adapt and adjust themselves in order to best possibly represent the objects of interest in an image. This paper briefly introduces a framework for Agent Based Image Analysis (ABIA) and presents our first research results.
\end{abstract}

\section{INTRODUCTION}

\subsection{OBIA for VHR image data analysis}

Very high resolution (VHR) remote sensing data and ObjectBased Image Analysis (OBIA) have influenced the remote sensing community since the beginning of the millennium. Although the latter builds on several older concepts and methods, such as image segmentation and knowledge based image interpretation, the particular combination of these concepts allows concepts that are common in Geography and other spatial disciplines, such as multi-scale analysis (Burnett and Blaschke 2003), to be applied. Meanwhile OBIA is an accepted paradigm for image analysis in the remote sensing community (Blaschke et al. 2014), which goes beyond analysing spectral statistical parameters. OBIA allows further object properties such as shape and spatial context to be incorporated (Benz et al. 2004, Blaschke 2010). Nevertheless, due to the scene complexity imaged with VHR data, complex image processing tasks as a kind of 'geo-intelligence' is necessary in order to draw on the full advantage of these data when applying methods of OBIA (Hay and Blaschke 2010).

While OBIA was regarded to be inextricably linked to image segmentation in the first years, since the segments provide the building blocks of object based image analysis (Hay and Castilla, 2008; Lang, 2008), it is meanwhile accepted that an optimum segmentation can only be achieved iteratively and knowledge-based, and that it is strongly bound to the application task and the data used (Baatz et al. 2008). Eventually, segments depict regions that ideally represent the objects of interest in the image of concern. Thus, the additional spatial information contained in objects is of even greater advantage than spectral per-object statistics (Benz et al., 2004; Hay and Castilla, 2008). It has been frequently claimed that this spatial dimension (shape, distances, neighbourhood, topologies, etc.) is crucial to OBIA methods. Authors increasingly recognize that the real potential lies in the intelligence and the chance to formulate user knowledge as 'rule sets' (Hofmann et al. 2011, Belgiu et al. 2014, Lang et al. 2014). However, the creation of robust, object-based solutions for automated image analysis of a set of images or even large image archives still remains extremely challenging (Pinz 2005, Walker and Blaschke 2008, Hofmann et al. 2011, Laliberte and Rango 2011, Kohli 2013). The highly complex content of VHR image data and the hardly predictable variability of the objects' qualities ultimately reduce the robustness and transferability of OBIA rule sets used for image classification. Fully automated, transferable and robust methods of remote sensing image analysis are not in sight yet. Moreover, either the rule sets or the objects' shape or even both need to be manually adapted in order to achieve acceptable results for a variety of images. Against this background, recent developments have paid more attention to the genericity of solutions (Novack et al. 2014) and the reusability of knowledge that has been used to create the complex rule sets (Arvor et al. 2014). However, in order to overcome the limited transferability of OBIA rule sets, we investigate the coupling, extension and integration of OBIA with principles and methods from the agent-based paradigm. In particular, this paper outlines a framework for Agent Based Image Analysis (ABIA), which extends the existing OBIA concepts and methods by those from the agent-based paradigm 
(Hofmann et al. 2015) and demonstrates our current implementations and first results achieved.

\subsection{From object-based to agent-based image analysis}

In OBIA, image analysis usually starts with a more or less arbitrary segmentation of the input data to generate a hierarchical net of image objects. These initially created image objects are then classified, or at least labelled with feature values, intrinsic for each object. Then, an iterative process of selective segmentation improvements and re-classifications begins, and ceases as soon as satisfactory results have been achieved. The criteria to define a current subset to operate on can be spatial, non-spatial, or both (Baatz et al. 2008, Lang 2008). A rule set in this context stores all processing steps, their procedural sequences and sub-sequences in order to re-apply them whenever necessary. Thus, a rule set can be seen as a kind of program written in a so-called Domain Specific Language (DSL), which uses linguistic elements and concepts of the application domain (Hudak 1996). A well-known example for such a DSL in the context of OBIA is the Cognition Network Language (CNL), which is implemented in the software eCognition (Athelogou et al. 2007). CNL provides two principle types of rules, which either calculate values or change the objects' shape and classification rules. While the former is self-explanatory, the latter either selects and processes a subset of objects based on defined criteria, or assigns objects to classes based on defined classification rules. The classification rules can be sample-based or explicit, and can be crisp or fuzzy, or incorporate a-priori probabilities. Additionally, classification rules can follow hierarchical (fuzzy) classification schemes. Fuzzy classification schemes describe each class as a fuzzy set based on fuzzy membership functions and their fuzzy-logic combinations (Benz et al. 2004). Each classification scheme reflects the object classes' remote sensing ontology, that is, their appearance in the image data at hand (Belgiu et al. 2014). Nevertheless, designing a rule set is labour intensive and to a certain degree subjective (Arvor et al. 2014, Belgiu et al. 2014). Its reusability can be limited once the input data changes (Hofmann et al. 2011). Against this background a conceptual framework for Agent Based Image Analysis (ABIA) has been introduced (Hofmann et al 2015), which describes two alternative approaches: (1) the agent-based, autonomous adaptation of OBIA rule sets, and (2) the evolvement of image objects to image object agents. Both approaches intend to allow a flexible reaction of rule sets and image objects on varying image data in order to produce similar classification results. In the present paper we briefly outline the conceptual framework, and present some simple and exemplary implementations thereof, together with some preliminary results. Finally we discuss the results and suggest future work to improve both the concepts and the results.

\section{SOFTWARE AGENTS AND MULTI AGENT SYSTEMS}

\subsection{Software agents}

According to the agent-based paradigm, software agents are flexible and capable of acting autonomously in complex and even unknown environments. Each agent is provided with sensors and effectors in order to interact with its environment and to achieve predefined goals. Thus, they are widely used in robotics and similar application fields. Several software agents can be combined in order to build a Multi Agent System (MAS). In a MAS software agents can interact, communicate, collaborate or negotiate among themselves in order to achieve individual or common goals. In such a system, each agent can have a specific role, which equips it with role-dependent abilities and goals. However, in a MAS, each software agent decides individually and based on its own strategy, when and how to act in a particular situation (Fig. 1).

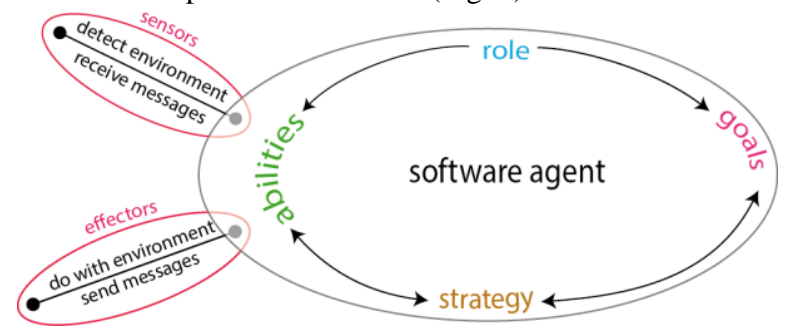

Figure 1. Principle components of individual software agents. Source: Hofmann et al. (2015).

These fundamental abilities have been shown to allow agentbased systems to deal with complex and unpredictable situations, as well as with incomplete information in a much more flexible and robust manner compared to conventional systems (Wooldridge and Jennings 1995, Jennings 2000).

\subsection{Multi agent systems in GIS and image analysis}

A common application of MAS in GIScience is agent based modelling. Agent Based Models (ABMs) simulate the behaviour of complex systems, such as societies or ecosystems. In Geography and related spatial sciences ABMs are used to simulate the evolvement and development of spatial patterns. During the simulation process the assumed behaviour of individuals interacting with their environment is modelled. Typical applications are the simulation of land use change or ecosystems and their spatial patterns (Parker et al. 2003, Macal and North 2005, Brown et al. 2005, Koch 2007, Marceau and Moreno 2008, Yu and Peuquet 2009).

Software agents are also widely applied in robotics. Similar to ABMs, individual software agents can be aggregated to Agent Based Systems (ABS) in order to control or maintain complex processes in industry. In this context ABS are increasingly used for process automation, which requires a high level of robustness and flexibility (Fazel Zarandi and Ahmadpour 2009, Göhner 2013).

However, in image analysis the usage of software agents or MAS is not very common yet. Bovenkamp et al. (2004) and Rodin et al. (2004) both describe a similar approach for object detection in Intra Vascular Ultra Sound (IVUS) images for medical image analysis. Samadzadegan et al. (2009, 2010) report on the application of ABS for building detection in LiDAR data. Mahmoudi et al. (2013) describe an ABS designed to improve OBIA classification results in urban areas. All these approaches have in common the distribution of different tasks of object recognition among respectively defined agents, that is, to parallelize image analysis using software agents. The agents simultaneously operate on an image and share their (intermediate) results. However, all these approaches focus on the potential of software agents to parallelize complex image processing tasks. The improved results are briefly presented by the authors.

\section{CONCEPTUAL FRAMEWORK FOR ABIA}

In contrast to the aforementioned agent-based approaches for image analysis, the conceptual framework for Agent Based Image Analysis (ABIA) outlined by Hofmann et al. (2015) 
focuses on the flexibility of ABS in order to increase the robustness of OBIA methods. Thus, it combines the ability of OBIA for a spatially detailed and semantic rich image analysis with the flexibility and robustness of ABS, aiming at increasing the reusability of OBIA rule sets. Since, in principle, both the rule sets and the image objects can adapt to changing image data, two alternative approaches were described by Hofmann et al. (2015). (1) Similar to ABS in robotics and process control, software agents automatically adapt existing rule sets according to varying image data: Software agents can autonomously change OBIA processing and classification rules. (2) Image objects evolve to image object agents: Each image object can autonomously adapt itself in order to best possibly fulfil its own classification criteria independent of the underlying image data. This approach has similarities to the concept of patch-based cellular automata, as introduced by Wang and Marceau (2013).

In both approaches the ontology of the image content plays a central and regulating role, since it acts as the foundation for the rule set and the object classes defined in the rule set with all their semantic constraints. The class descriptions, that is, the classification rules, can even be directly translated from the ontology (Belgiu et al. 2014). However, the ontology describes the object classes and their semantics independently from any underlying image data, and must not be violated by any agentbased adaptations.

Another aspect that impacts the agents' behaviour is the achieved quality during or after adaptation. Quality by definition reflects the minimum properties of an acceptable classification result (ISO 19157:2013). In remote sensing this refers to correctness and completeness. Both are measured by comparing the classification result with a reference classification that is assumed to be true (Congalton and Green 2009). However, a complete conformity between reference data and classification results is often not achievable (Hofmann and Lohmann, 2007, Albrecht et al. 2010). Thus, evaluating the classification quality should rather be measured according to the user requirements in terms of the required correctness and completeness. Nevertheless, in both ABIA concepts the minimum quality to be achieved has to be defined as a goal.

\subsection{MAS to autonomously adapt existing rule sets}

A MAS designed to change existing rule sets consists at least of one or more Rule Set Adaptation Agents (RSAA). Since the number of potential rule set adaptations can be as numerous as the number of rules and parameters, there is no limit for the number of RSAAs being organized in such a MAS. However, each ABIA-MAS must balance its activities between ontology conformity and quality requirements. That is, only rule set adaptations not violating the ontology and leading to best achievable quality are permitted. In the framework presented by Hofmann et al. (2015), this balancing and evaluation act is performed by one or several dedicated control agents (CAs). Their goals are designed to prohibit adaptations that lead to a contradiction between ontology and rule set, and to achieve a minimum quality, usually at least the quality of the initial classification the rule set was created for. Consequently, the adaptation process is evolutionary, whereby RSAAs and CAs try to find the best adaptations in order to achieve their common goals. During this process, CAs inform RSAAs whether their intended actions are allowed or not, and to what degree their adaptations would be successful in terms of goal achievement. In the outlined MAS, each RSAA is designed to adapt specific elements of a given rule set, which means to adjust parameters, to change classification rules, and to add or remove processing rules, etc. Depending on the type of adaptation, RSAAs can be sub-divided into diverse types of RSAAs. However, each RSAA has individual sensors, effectors, goals and strategies. Additionally, each agent should be provided with a learning mechanism and a cost model allowing it to focus on the most promising adaptation actions (see Fig. 2).

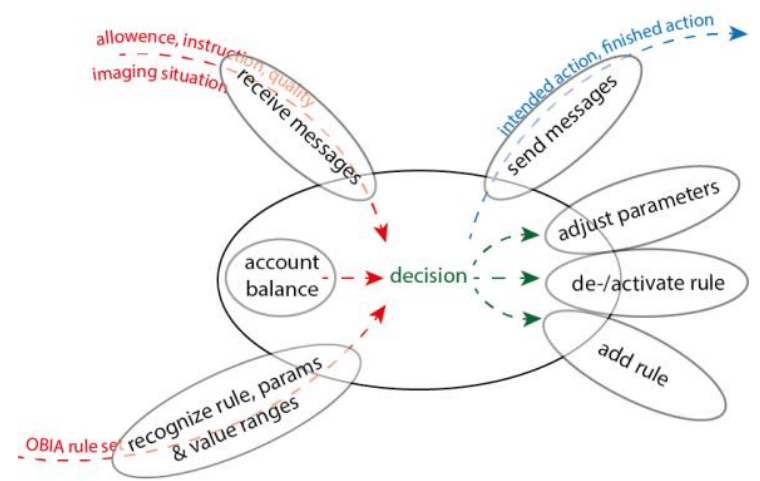

Figure 2. Principle design of a RSAA. Source: Hofmann et al. (2015).

\subsection{Hierarchical net of Image Object Agents}

Alternatively to a MAS consisting of RSAAs and CAs, image objects aka segments can evolve to image object agents (IOAs) and together build a MAS, organized as a (hierarchical) net of IOAs. Similar to the hierarchical net of image objects as outlined in Benz et al. (2004), each IOA is connected with its sub-, super- and neighbour agents. This allows each IOA to request the current status of the connected IOAs, and to provide information about its own status. However, the ABIA concept extends the OBIA approach by adding the ability of each IOA to process itself, and to interact, collaborate and to negotiate with other IOAs. Additionally, IOAs have individual goals or share goals with other IOAs, motivating each IOA to execute or to not execute certain actions. If a cost model is additionally integrated, each IOA aims at reducing costs, too. Implementing learning mechanisms allows each IOA to avoid the repetition of unsuccessful actions, and to focus on the most promising strategies. If an IOA is a member of at least one class, one of its goals will be to fulfil the classification criteria as good as possible, that is, to match the class antetype as described in the ontology and according class definition. Further goals can be to achieve pre-defined quality criteria, as outlined in the section before, or to avoid classification ambiguities and uncertainties. For a MAS built up by IOAs, as depicted here, it can also be sensible to encapsulate global tasks, such as executing the classification, controlling the global quality, prioritising tasks or classes, etc. in one or more CAs.

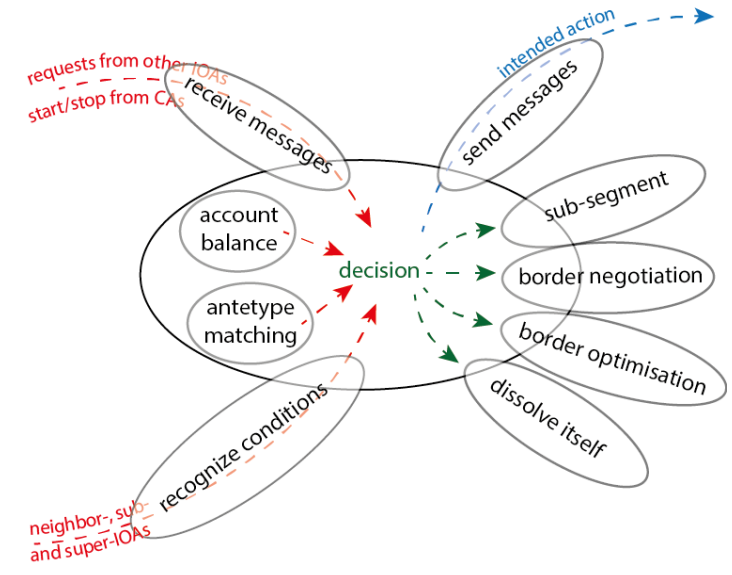


Figure 3. Principle design of an IOA. Source: Hofmann et al. (2015).

\section{CURRENT IMPLEMENTATIONS}

Both of the described approaches of ABIA have been implemented in different environments. The IOA approach as outlined in section 3.2 has been implemented twice: One implementation is based on eCognition and its CNL and has already been realized and documented in Hofmann et al. (2015). The second implementation is introduced here. It integrates existing non-commercial tools for $\mathrm{ABM}$, image processing and GIS with self-coded software. The necessary image processing methods, object property calculations, and fuzzy classification mechanisms, as well as the necessary methods to exchange data between the tools and components (Fig. 4) were implemented by the authors.

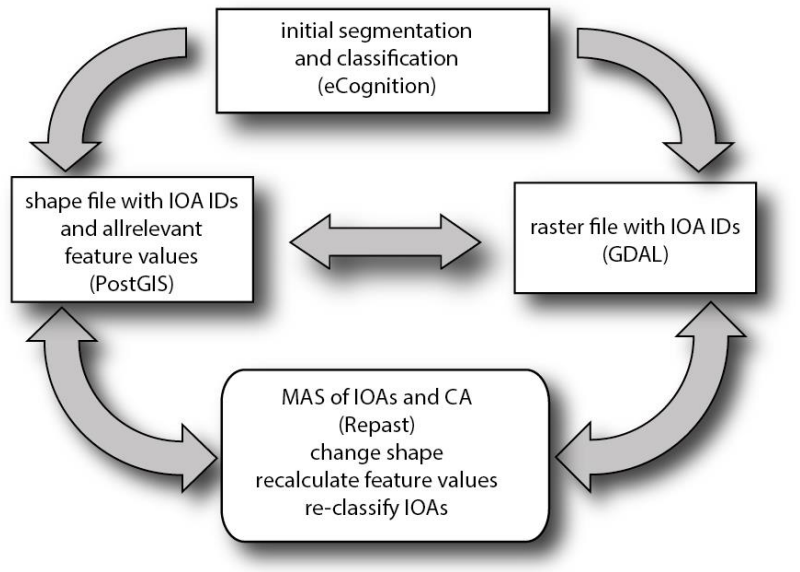

Figure 4. Principle adaptation workflow and components of an ABIA MAS consisting of IOA.

For the realisation of the RSAA approach as outlined in section 3.1, Repast was used for the agent-based processing, and eCognition for the execution of the adapted OBIA rule set. Additionally, a DSL was developed, which allows the specific agents for this approach to be very easily defined (Lettmayer, 2015). Recently, none of the implementations contains a learning method, yet, whereas the integrating implementation of the IOA approach uses a simple cost model.

All approaches were developed based on a simple scenario consisting of a subset of an orthorectified VHR airborne scene from Weilheim, Upper Bavaria, Germany, taken in May 2010. The orthoimage together with a DSM were derived from a stereo pair of the RGB-bands captured by an UltraCam XP (www.ultracamx.com) using software from SimActive (www.simactive.com). The spatial resolution of the orthoimage is $8 \mathrm{~cm}$, and that of the DSM is $35 \mathrm{~cm}$. The radiometric resolution of the optical data is 8bit. Slope and slope of slope (change of slope) were calculated from the DSM per pixel and expressed in degrees. The subset under investigation has a size of 1311 x 869 pixels. The initial segmentation and classification were performed using eCognition, whereby the rule set was designed to delineate roofs and different roof types ("saddled", "flat" and "other"). The CNL rule set consists of an initial multi-resolution segmentation, which uses the R, G, and B band of the image together with the DSM and slope values as input data, and parameters as outlined in Tab. 1.

\begin{tabular}{|c|c|c|c|c|}
\hline Level & Method & scale parameter $^{1)} /$ size $^{2)}$ & shape & compactness \\
\hline 1 & Chessboard & $1^{2)}$ & - & - \\
2 & MRS & $150^{1)}$ & 0.2 & 0.5 \\
\hline
\end{tabular}

Table 1. Segmentation parameters

It then classifies each image object according to a fuzzy class hierarchy describing the different roof types (see Appendix 1), which is based on an appropriate ontology. The initial parameterisation of the segmentation leads to a general oversegmentation of the scene and an incomplete detection of roofs (see Fig. 5).

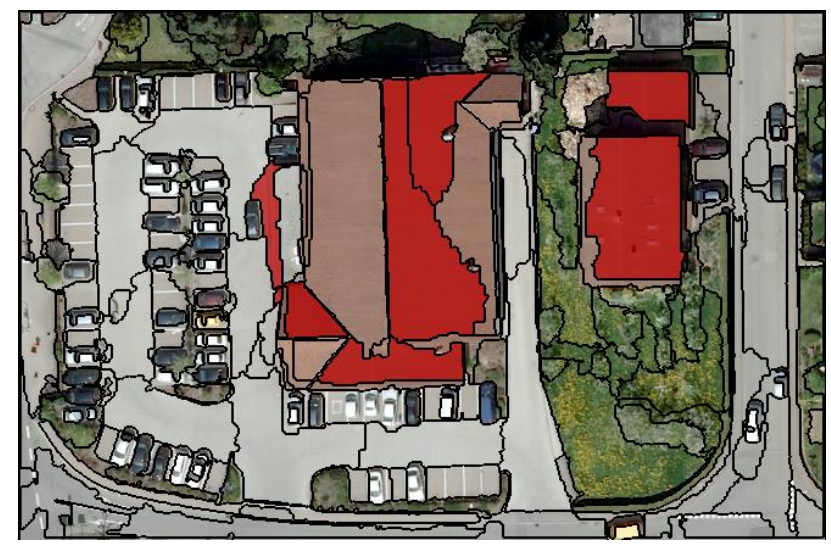

Figure 5. Initial segmentation and classification result.

\subsection{Agent-based adaptation of existing rule set}

As outlined in section 3.1, the MAS for this approach consists of at least one RSAA and one CA. We decided to reduce the technical complexity of the MAS to a minimum in order to demonstrate the principle feasibility,. Consequently, our current configuration of this MAS consists of exactly one RSAA and one CA. The MAS (Fig. 6) tries to adjust the so-called scale parameter of the multi-resolution segmentation (Baatz \& Schäpe, 2000).

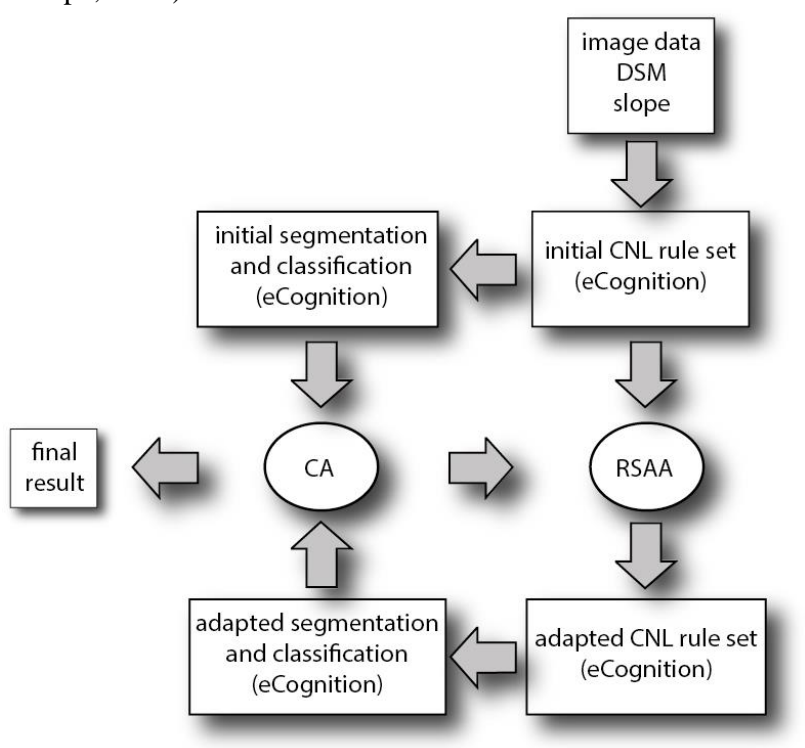

Figure 6. Principle adaptation workflow and components of an ABIA MAS consisting of RSAA.

Goal of the MAS is to adjust the scale parameter in a way that the resulting segments delineate house objects as good as possible. The latter is evaluated through their individual degree of membership to one of the "roof"-type sub-classes: the higher 
the membership degree, the better they delineate a house object. To evaluate the whole scene, the mean best membership value of all "roof"-objects to one of these roof classes is calculated. Unclassified objects remain disregarded. The higher the mean best membership, the more the MAS has achieved its goal. In the present example we accept a classification result that produces at least a mean best membership of 0.7. That is, once this threshold is achieved (within a pre-defined number of iterations aka ticks), the MAS will stop the adaptation process.

RSAA and CA were defined using the so-called Agent Definition Language (ADL), which is a DSL especially designed for agent-based autonomous OBIA rule set adaptation (see Lettmayer, 2015 for details). They are translated in Java code and executed in Repast (Collier, 2003) in order to adapt the XML-coded CNL rule set. In each adaptation tick the adapted rule set is automatically executed with eCognition, and the resulting mean best membership value is evaluated by the CA. In case the goal is not achieved yet, the CA triggers the RSAA to adapt the scale parameter again, and the next adaptation tick starts (Fig. 6).

\subsection{Self-organising net of Image Object Agents}

In principle, IOAs and their behaviour can be implemented in different environments and configurations. The basic idea in the example given is to provide each IOA with the goal to become a best possible member of one of the roof-type classes, and to individually decide on optional actions in order to change its own shape. Hofmann et al. (2015) demonstrate how this can be realised in eCognition using its CNL. However, eCognition does not allow direct access to single pixels of objects, which is essential for autonomous IOA shape adjustment. Additionally, the approach of Hofmann et al. (2015) does not contain a cost model. Thus, we decided to implement the IOA approach by integrating existing GIS and tools: PostGIS (Lijing \& Jing, 2010), and GeoJSON, (Butler et al., 2008), image processing tools, here: GDAL (http://www.gdal.org/), and tools for ABM, here: Repast. Initially segmented and classified objects are processed in this environment. Each IOA can access its own pixels, which allows it to exchange its border pixels with neighbouring IOAs, and vice versa. Currently, only five neighbouring border pixels can be exchanged at once per IOA and tick, due to the still complex exchange mechanism. The latter currently uses a CSV file that stores all exchanging IOAs and their exchangeable pixels. In order to avoid a deterioration of neighbouring IOAs, each IOA prefers those neighbours for pixel exchange that have the lowest membership degree for one of the roof-type classes. The recent cost model decreases an IOA's account by two currency units for each action. If the performed action improved the IOAs class membership, it gets three currency units back, which "rewards" the action's success. Our database stores the evolution of each IOA, namely the shape and all feature values of each IOA at each tick.

\section{RESULTS}

While for the implemented RSAA approach and the IOA approach implemented according to Hofmann et al. (2015) an improvement is observable, this is not the case for the IOA approach using our own integrated environment. The reasons for the latter will be discussed in the next section.

As Fig. 8 indicates, the autonomous adaptation of the scale parameter for the multi-resolution segmentation improves the initial classification results, that is, the mean membership of all house IOAs to one of the roof type classes. While the initial value was at 0.14 , it improved to 0.56 after 100 ticks, which is still below the aspired minimum value of 0.7 (Fig. 7 and 8). The results are similar to those achieved by Hofmann et al. (2015). Nevertheless, parts of the big central building in the lower left corner and the upper right corner were not connected to the main object. Similarly, the garage bordering the smaller building in the central-right part of the image was neither delineated nor classified as a roof-type object (false negatives). Vice versa, the building's shape at its northbound border was wrongly classified as a roof-type object (false positive). The reasons for these misclassifications are discussed in the next section.

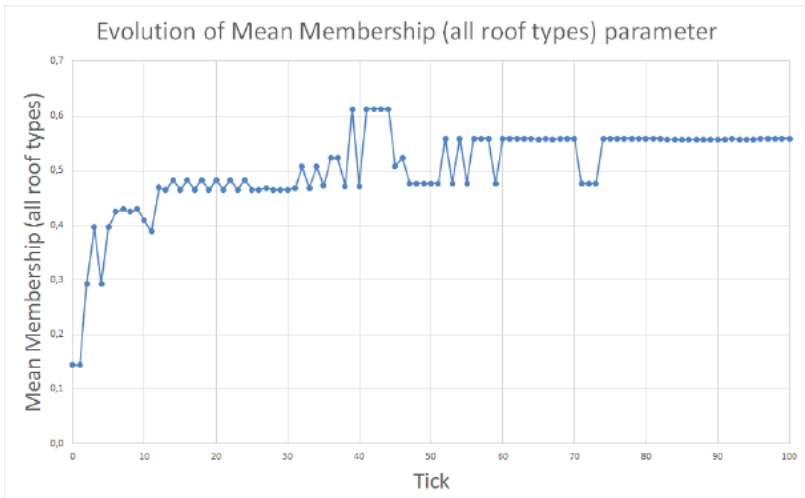

Figure 7. Development of the mean membership of all roof-type IOA for one of the roof-type classes when applying the ABIA MAS consisting of RSAA.

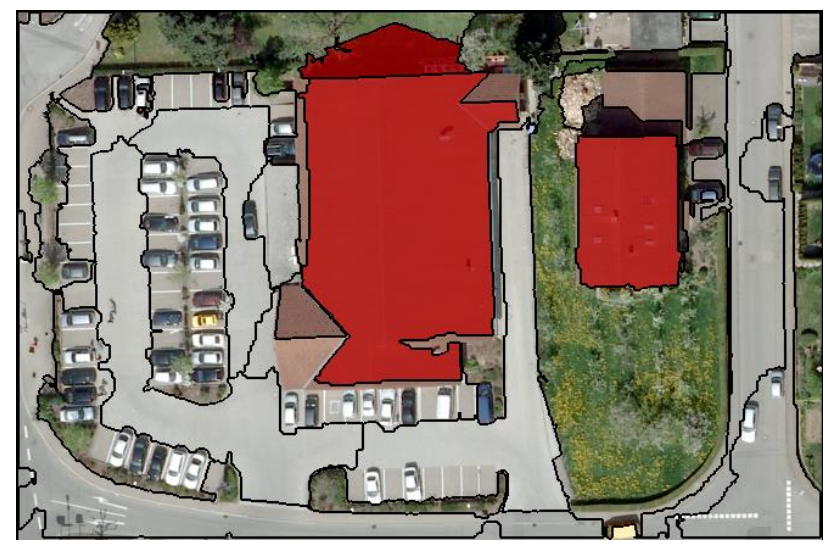

Figure 8. Final segmentation and classification result applying the ABIA MAS consisting of RSAA.

In contrast, for our integrated solution the mean membership of all house IOAs to one of the roof type classes decreases (see Fig. 9).

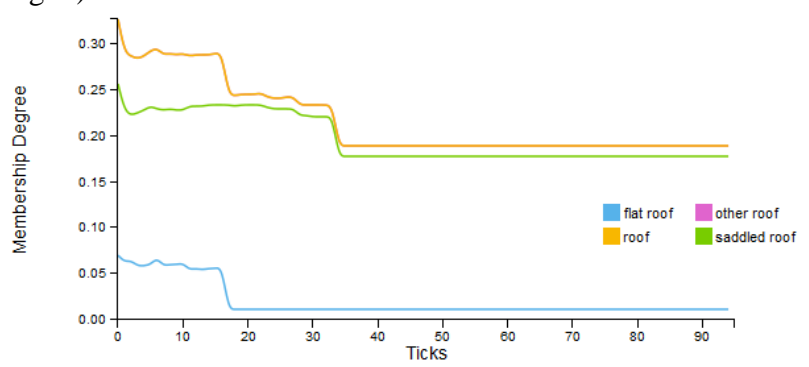

Figure 9. Development of mean membership degrees for rooftype classes applying the integrated IOA approach.

Additionally, even after 100 ticks almost no change of the objects' shape is observable (see Fig 10 in comparison with Fig. $5)$. 


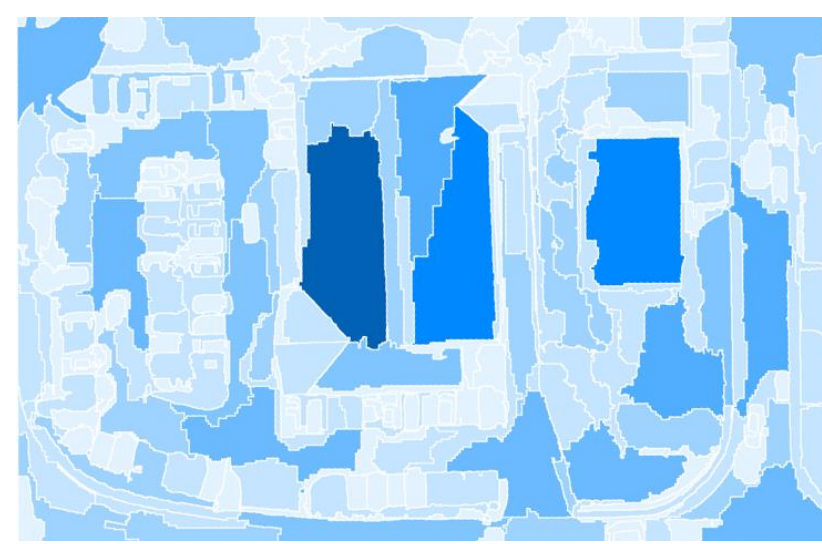

Figure 10. Final segmentation result after applying the ABIA MAS consisting of IOA for 100 ticks.

Nevertheless, we were able to demonstrate that it is possible in principle to realise a MAS of IOAs according to the conceptual framework introduced in Hofmann et al. (2015), and wherein neighbouring IOAs are capable of exchanging border pixels following individual goals, that is, to reduce transaction costs and to improve their own membership to one of the desired classes.

\section{CONCLUSIONS AND DISCUSSION}

We could demonstrate that the implementation of the conceptual framework for ABIA and the different adaptation approaches as described in Hofmann et al. (2015) are feasible in principle. For the example data used we could also show that besides the already demonstrated IOA solution as implemented with CNL in eCognition, rule set adaptation with RSAAs is also capable of improving initial OBIA segmentation and classification results. In this context it turned out that the development and usage of a DSL supports the creation and design of ABIA MASs. That is, the necessary knowledge (here: about JAVA, Repast and eCognition) to create an ABIA MAS can be reduced to a minimum, while the user can focus on the design of necessary software agents. Thus, we plan to further develop and extend the developed ADL in order to make it applicable for the IOA approach also. Nevertheless, the results are not perfect. A potential reason for the false negatives mentioned in section 5 is the already "perfect" shape achieved, when the central parts of the bigger building evolve to a rooftype object. Since the unlinked outer parts are jutties, with locally different slopes and shapes, integrating them with the main part would lead to a reduction of the membership to one of the roof-type classes. The latter would contradict the MAS's goal. For the same reason, the garage roof cannot merge with its bordering shade area or even the bordering building. Thus, leaving the garage unclassified but completely delineating the neighbouring building leads to an overall better result in terms of membership degrees. The detectable false positive area at the upper border of the central building is in fact the shade of this building casted on a neighbouring tree. Since the slope and elevation of the tree is similar to that of buildings, and the casted shade has the building's shape, this segment already fulfils the classification criteria of a roof-type class. Nevertheless, its degree of membership to the class "saddled roof" is only at $\mu=0.15$. Those of the central building and the smaller right-hand building are at $\mu=0.94$ and $\mu=0.99$. However, introducing an explicit distinct class "shade" to the ontology and the class hierarchy could easily solve this problem.

The reasons for the rather disappointing results of our integrated IOA approach are manifold. One is certainly given by the recently missing alternative actions each IOA can execute. Such an alternative action could be, for example, merging with neighbouring IOAs. Especially if the image resolution is as extremely high as in the example given $(0.08 \mathrm{~m})$, merging could certainly accelerate the adaptation process. With this high resolution, exchange of (five neighbouring) border pixels has almost no impact on the IOAs' shape. Thus, the amount of exchangeable pixels (e.g. along the whole border or even inside a to-be-defined buffer) should be increased. Nevertheless, more research with lower resolutions is necessary. Similarly, the presented RSAA approach has no cost model implemented yet, since this is not sensible with only one acting option (adjust the scale parameter) However, if alternative actions were implemented, which is planned, a cost model for RSAAs is also necessary. Last but not least, none of the implemented MAS contains agents with the ability to learn. Having a learning method available for each agent could further support them in their adaptation and optimisation process. Additionally, future research will take into account the possibility of parallelizing ABIA activities.

Besides the presented approach for using IOAs, they could also be used in ABM, as described in section 2.2. In combination with remote sensing data this would offer the opportunity to simulate and validate an $\mathrm{ABM}$ simultaneously based on remote sensing data. In combination with the used ADL, even non-experts could easily perform ABM.

\section{ACKNOWLEDGEMENTS}

The research presented was funded by the FWF basic research project ABIA, grant number FWF P25449. We also thank Thomas Strellen (TS Photogrammetrie \& Geoinformatik, www.photogeo.de) for providing us with the UltraCam XP image data and SimActive (www.simactive.com) for supporting us in the generation of the DSM.

\section{REFERENCES}

Athelogou, M., et al., 2007. Definiens Cognition Network Technology - A Novel Multimodal Image Analysis Technique for Automatic Identification and Quantification of Biological Image Contents. In: Frischknecht, F., Shorte, S.L., (Eds.): Imaging Cellular and Molecular Biological Functions, Springer. Berlin, Heidelberg. pp. 407-421.

Albrecht, F., Lang, S. and Hölbling, D., 2010. Spatial Accuracy Assessment of Object Boundaries for Object-Based Image Analysis. The International Archives of the Photogrammetry, Remote Sensing and Spatial Information Sciences, XXXVIII4/C7.

Arvor, D., Durieux, L., Andrés, S. and Laporte, M.-A., 2013. Advances in Geographic Object-Based Image Analysis with ontologies: A review of main contributions and limitations from a remote sensing perspective. ISPRS Journal of Photogrammetry and Remote Sensing, 82 (0), pp. 125-137.

Baatz, M., Hoffmann, C. and Willhauck, G., 2008. Progressing from object-based to object-oriented image analysis. In: Blaschke, T. Lang, S. and Hay, G. (eds.) Object-Based Image Analysis: Spatial Concepts for Knowledge-Driven Remote Sensing Applications. Berlin, Heidelberg: Springer.

Belgiu, M., Hofer, B. and Hofmann, P., 2014a. Coupling formalized knowledge bases with object-based image analysis. Remote Sensing Letters, 5 (6), pp. 530-538. 
Benz, U. C. et al., 2004. Multi-resolution, object-oriented fuzzy analysis of remote sensing data for GIS-ready information. ISPRS Journal of Photogrammetry and Remote Sensing, 58, pp. 239-258.

Blaschke, T. and Strobl, J., 2001. What's wrong with pixels? Some recent developments interfacing remote sensing and GIS. GIS - Zeitschrift für Geoinformations-systeme, 14 (6), pp. 1217.

Blaschke, T. et al., 2014. Geographic Object-Based Image Analysis - Towards a new paradigm. ISPRS Journal of Photogrammetry and Remote Sensing, 87, pp. 180-191.

Bovenkamp, E. G. P. et al., 2004. Multi-agent segmentation of IVUS images. Pattern Recognition, 37(4), 647-663.

Brown, G. D. et al., 2005. Spatial process and data models: Towards integration of agent-based models and GIS. Journal of Geographical Systems, 7, pp. 25-47.

Burnett, C. and Blaschke, T., 2003. A multi-scale segmentation/object relationship modelling methodology for landscape analysis. Ecological Modelling, 168 (3), pp. 233-249.

Butler, H., Daly, M., Doyle, A., Gillies, S., Schaub, T., Schmidt, C., 2008. The GeoJSON format specification. Rapport technique, 67.

Collier, N., 2003. Repast: An extensible framework for agent simulation. The University of Chicago's Social Science Research, 36, 2003.

Congalton, R. G. and Green K., 2009. Assessing the Accuracy of Remotely Sensed Data-Principles and Practices. Boca Raton, FL CRC Press, Taylor \& Francis Group.

Cracknell A. P., 1998. Synergy in remote sensing - what's in a pixel? International Journal of Remote Sensing, 19 (11), pp. 2025-2047.

Fazel Zarandi, M. H. and Ahmadpour, P., 2009. Fuzzy agentbased expert system for steel making process. Expert Systems with Applications, 36, pp. 9539-9547.

Göhner, P., 2013. Agentensysteme in der Automatisierungstechnik. Berlin, Heidelberg: Springer.

Hay, G. J. and Blaschke, T., 2010. Special issue: geographic object-based image analysis (GEOBIA). Photogrammetric Engineering and Remote Sensing, 76 (2), pp. 121-122.

Hay, G. J. and Castilla, G., 2008. Geographic Object-Based Image Analysis (GEOBIA): A new name for a new discipline. In: Blaschke, T. Lang, S. and Hay, G. (eds). Object-Based Image Analysis: Spatial Concepts for Knowledge-Driven Remote Sensing Applications. Berlin, Heidelberg: Springer.

Hofmann, P. and Lohmann, P., 2007. A strategy for quality assurance of land-cover/land-use interpretation results with faulty or obsolete reference data. The International Archives of the Photogrammetry, Remote Sensing and Spatial Information Sciences, XXXVI (1/W51).

Hofmann, P., Blaschke, T. and Strobl, J., 2011. Quantifying the robustness of fuzzy rule sets in object-based image analysis. International Journal of Remote Sensing, 32 (20), pp. 73597381.
Hofmann, P., et al. 2015. Towards a framework for agent-based image analysis of remote-sensing data. International Journal of Image and Data Fusion, 6 (2), pp. 115-137.

Hudak, P., 1996. Building domain-specific embedded languages. ACM Computing Surveys, 28 (4).

International Organization for Standardization, 2013. ISO 19157:2013. Geographic information - Data quality.

Jennings, N. R., 2000. On agent-based software engineering. Artificial Intelligence, 117, pp. 277-296.

Koch, A., 2007. Geovisualisierung durch Geosimulation. Visuelle Repräsentation humangeographischer Phänomene durch Agentenbasierte Geosimulation. In: Tzschaschel, S., Wild, H., Lentz, S. (eds.) Visualisierung des Raumes. Karten machen - die Macht der Karten. Forum ifl, pp. 191-202.

Kohli, D. et al., 2013. Transferability of Object-Oriented Image Analysis Methods for Slum Identification. Remote Sensing, 5, pp. 4209-4228.

Laliberte, A. S. and Rango, A., 2011. Image Processing and Classification Procedures for Analysis of Sub-decimeter Imagery Acquired with an Unmanned Aircraft over Arid Rangelands. GIScience \& Remote Sensing, 48 (1), pp. 4-23.

Lang, S., 2008. Object-based image analysis for remote sensing applications: modelling reality - dealing with complexity. In: Blaschke, T. Lang, S. and Hay, G. (eds). Object-Based Image Analysis: Spatial Concepts for Knowledge-Driven Remote Sensing Applications. Berlin, Heidelberg: Springer.

Lang, S. et al., 2014. Geons - domain-specific regionalization of space. Cartography and Geographic Information Science, 41 (3), pp. 214-226.

Lettmayer, P., 2015. Domain specific language for agent-based image analysis. Master thesis, University of applied sciences Salzburg.

Lijing Z; Jing Y, 2010. Management methods of spatial data based on PostGIS. In Circuits, Communications and System (PACCS), 2010 Second Pacific-Asia Conference on , Vol.1, No.1, pp.410-413, 1-2 Aug. 2010

Macal C. M. and North, M. J., 2005. Tutorial on agent-based modeling and simulation. In: Kuhl M. E., Steiger N. M., Armstrong F. B. and Joines J. A. (Eds.), 37th Winter Simulation Conference, 2005 Orlando, FL, USA.

Mahmoudi, F. T., Samadzadegan F. and Reinartz, P., 2013. Object oriented image analysis based on multi-agent recognition system. Computers \& Geosciences, 54, pp. 219-230.

Maillot, E. N. and Thonnat, M., 2008. Ontology based complex object recognition. Image and Vision Computing, 26 (1), pp. 102-113.

Motik, B., Patel-Schneider, P. F. and Grau, B. C., 2009. Owl 2 web ontology language direct semantics. W3C recommendation, 27.

Novack, T., Kux, H., Feitosa, R. Q. and Costa, G. A. O. P., 2014. A knowledge-based, transferable approach for blockbased urban land-use classification. International Journal of Remote Sensing, 35(13), pp. 4739-4757. 
Parker, D. C. et al., 2003. Multi-agent systems for the simulation of Land-Use and Land-Cover change: A review. Annals of the Association of American Geographers, 93 (2), pp. 314-337.

Pinz, A., 2005. Object Categorization. Foundations and Trends in Computer Graphics and Vision, 1 (4), pp. 255-353.

Rodin, V. et al., 2004. An immune oriented multi-agent system for biological image processing. Pattern Recognition, 37 (4), pp. 631-645.

Samadzadegan, F., Schenk, T. and Mahmoudi, F. T., 2009. A multi-agent method for automatic building recognition based on the fusion of Lidar range and intensity data. Joint Urban Remote Sensing Event, 2009, 20-22 May 2009, Shanghai, China, 1-6.

Samadzadegan, F., Mahmoudi, F. T. and Schenk, T., 2010. An agent-based method for automatic building recognition from lidar data. Canadian Journal of Remote Sensing, 36 (3), pp.

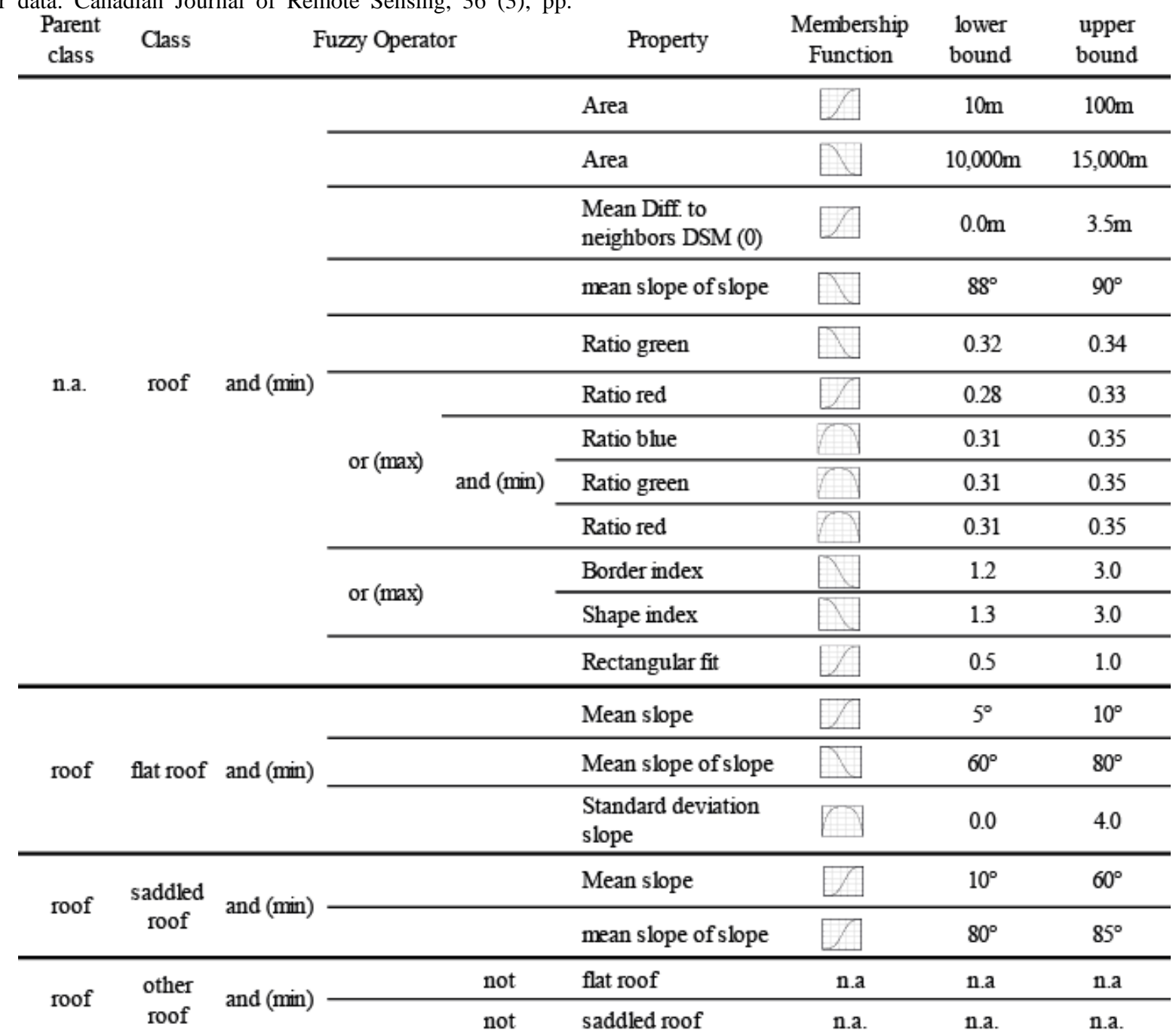

Appendix 1: Classes and class descriptions. Source: Hofmann et al (2015).
211-223.

Walker, J. S. and Blaschke, T., 2008. Object-based landcover classification for the Phoenix metropolitan area: optimization vs. transportability. International Journal of Remote Sensing, 29 (7), pp. 2021-2040.

Wooldridge, M. and Jennings, N. R., 1995. Intelligent agents: Theory and practice. Knowledge Engineering Review, 10 (2), pp. 115-152.

Yu, C. and Peuquet, D. J., 2009. A GeoAgent-based framework for knowledge-oriented representation: Embracing social rules in GIS. International Journal of Geographical Information Science, 23 (7), pp. 923-960.

\section{APPENDIX}

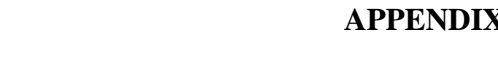

